\title{
Avaliação do perfil metabólico, nutricional e efeitos adversos de crianças com epilepsia refratária em uso da dieta cetogênica
}

\author{
Assessment of serum biochemistry, nutritional status \\ and adverse effects of children with refractory \\ epilepsy using the ketogenic diet
}

Sueli RIZZUTTI'

Ana Maria Figueiredo RAMOS²

Isa de Pádua CINTRA ${ }^{3}$

Mauro MUSZKAT ${ }^{4}$

Alberto Alain GABBAI ${ }^{5}$

RE S U M O

\section{Objetivo}

Avaliar os efeitos adversos, o perfil metabólico e o crescimento pôndero-estatural de crianças com crises epilépticas de difícil controle, as quais foram submetidas a dieta cetogênica.

\section{Métodos}

Selecionaram-se 23 pacientes na faixa etária de 2 até 17 anos com epilepsia de difícil controle medicamentoso, sendo $43,5 \%(n=10)$ do sexo masculino e 56,5\% $(n=13)$ do sexo feminino, provenientes do Setor de Neuropediatria da Disciplina de Neurologia da Universidade Federal de São Paulo. Foram submetidos a dieta cetogênica e acompanhados por um período mínimo de um ano. Dois pacientes não conseguiram manter a cetose por falta de adesão dos pais à dieta.

\section{Resultados}

Os efeitos adversos encontrados foram reversíveis, incluindo hiperlipidemia, obstipação (17,4\%), náuseas e vômitos $(43,4 \%)$, sonolência $(47,8 \%)$, infecções intercorrentes $(3,0 \%)$, recusa da dieta $(13,0 \%)$ e epistaxe

\footnotetext{
1 Doutoranda, Disciplina de Neurologia, Escola Paulista de Medicina, Universidade Federal de São Paulo. Rua Botucatu, 740, 04023-900, São Paulo, SP, Brasil. Correspondência para/Correspondence to: S. RIZZUTTI. E-mail: <surizzutti@ig.com.br>.

2 Nutricionista, Departamento de Pediatria, Universidade Federal de São Paulo. São Paulo, SP, Brasil.

3 Universidade Federal de São Paulo. São Paulo, SP, Brasil.

4 Departamento de Psicobiologia, Universidade Federal de São Paulo. São Paulo, SP, Brasil.

${ }^{5}$ Departamento de Neurologia e Neurocirurgia, Universidade Federal de São Paulo. São Paulo, SP, Brasil..
} 
(4,3\%). O crescimento pôndero-estatural não foi afetado, tendo o peso e a estatura seguido o percentil adequado.

\section{Conclusão}

A dieta cetogênica pode constituir-se em uma alternativa segura e efetiva para o tratamento de crianças com epilepsia refratária.

Termos de indexação: criança; dieta; epilepsia; lipídeos na dieta.

\section{A B S T R A C T}

\section{Objective}

The purpose of this research was to evaluate adverse events, serum biochemistry, growth and nutritional status of children with difficult-to-control seizures who were submitted to ketogenic diet.

\section{Methods}

Twenty-three patients aging from 2 to 17 years with refractory epilepsies, where $43.5 \%(n=10)$ were males and $56.5 \%(n=13)$ females from the Sector of Neuropediatrics, Discipline of Neurology of the Universidade Federal de São Paulo, were treated with the ketogenic diet and followed up for at least 1 year. Two patients were not able to achieve persistent ketosis either because they rejected the diets or their parents did not comply.

\section{Results}

Adverse events were all reversible and included hyperlipidemia, constipation (17.4\%), nausea and vomiting (43.4\%), drowsiness (47.8\%), intercurrent infections (3.0\%), diet refusal (13.0\%) and epistaxis (4.3\%). Growth was not affected in the short term and most heights remained within the normal centile lines.

\section{Conclusion}

The ketogenic diet can be considered a safe and effective treatment for children with intractable seizures.

Indexing terms: child; diet; effects; epilepsy; dietary fats.

\section{N T R O D U Ç Ã O}

A adoção da dieta cetogênica para o controle de pacientes epilépticos começou a se destacar durante a década de 20, na Clínica Mayo, nos Estados Unidos. O postulado original de Wilder mostrava que uma dieta rica em gordura e pobre em carboidratos poderia reproduzir a cetose e a acidose metabólica características do estado de jejum, permitindo-lhe a manutenção por período maior de tempo, num nível nutricional satisfatório ${ }^{1,2}$.

Na década de 1990, assistiu-se a uma progressiva retomada da dieta cetogênica no tratamento das epilepsias, mesmo diante dos avanços tecnológicos para a investigação e o tratamento das doenças neurológicas e do desenvolvimento concomitantemente ao uso de drogas antiepilépticas modernas ${ }^{3,4}$.
A dieta cetogênica tem sido um tratamento opcional, instituído no setor de Neuropediatria da Universidade Federal de São Paulo, há três anos. A experiência abordada, embora bastante recente, representa o fruto do trabalho dedicado de uma equipe multidisciplinar composta por psicóloga, nutricionista, assistente social e neurologista.

Neste contexto, desenvolveu-se um estudo para avaliar o perfil metabólico, nutricional, os efeitos adversos, como também, a eficácia do uso da dieta cetogênica em crianças com epilepsia refratária.

\section{MÉ T O D O S}

Foram selecionados 23 pacientes, na faixa etária de 1 ano e 6 meses a 17 anos, com epilepsia de difícil controle medicamentoso, provenientes 
do Setor de Neuropediatria - Disciplina de Neurologia, Universidade Federal de São Paulo (Unifesp). Como tratamento alternativo, ofereceu-se aos pais a opção da dieta cetogênica. As crises epilépticas foram classificadas de acordo com a Classificação Internacional das Crises Epilépticas ${ }^{5}$. Os pacientes da amostra foram selecionados por meio de avaliação médica, nutricional, psicológica e social, e submetidos à dieta, com um tempo de seguimento de 12 meses. Utilizou-se a proporção cetogênica ${ }^{6}$ de 4:1.

Durante um período mínimo de um ano, monitorou-se, prospectivamente, um grupo de pacientes com epilepsia de difícil controle, submetidos à dieta cetogênica, além de estudar o perfil metabólico, nutricional e os efeitos adversos.

Todos os pacientes foram acompanhados no Setor de Neuropediatria, por um período mínimo de seis meses de tratamento clínico com drogas antiepilépticas habituais e novas drogas, em regime de politerapia. Antes de serem incluídos no trabalho, os pacientes apresentavam uma freqüência maior do que três crises por semana.

Os pacientes passaram por exames metabólicos de controle antes do início da dieta e com repetição a cada três meses, entre eles: dosagem de colesterol e frações, triglicérides, ácido úrico, hemograma completo, dosagem de cálcio, dosagem de magnésio, sódio, cloro, potássio, glicemia, uréia, creatinina, gasometria venosa, eletroforese de proteínas, urina I, dosagem de cálcio e creatinina urinária. Também foram feitos ultrassonografia abdominal e eletrocardiograma a cada quatro meses. Após o término do estudo, procedeu-se à comparação dos resultados.

Este trabalho foi aprovado pela Comissão de Ética da Unifesp-EPM e os pais ou responsáveis pelos menores assinaram o termo de consentimento informado.

\section{Resposta clínica}

Elaborou-se uma tabela de freqüência das crises epilépticas para cada paciente, confrontando-a, em seguida, com a análise da freqüência de todos os tipos de crises de cada um, realizando, assim, um estudo comparativo e, por fim, utilizaram-se os seguintes critérios de Huttenlocher ${ }^{7}$ sobre resposta antiepiléptica:

- Excelente controle: controle completo das crises epilépticas;

- Muito bom controle das crises epilépticas: diminuição de mais de $90 \%$ das crises;

- Bom controle: diminuição de 50\% a 90\% das crises epilépticas;

- Controle regular: $<50 \%$ de diminuição no número de crises epilépticas;

- Ausência de efeito: sem mudança na frequência de crises;

- Negativo: aumento do número de crises.

Para comparar desistentes e aderentes quanto ao comportamento observado ao longo do tempo, aplicou-se o modelo de análise de variância com medidas repetidas. O pacote estatístico usado foi o R 2.0.1.

\section{RES U LTA D OS}

O grupo de pacientes com epilepsia de difícil controle foi constituído por 23 pacientes, com idade mediana de 8 anos, variando de 4 até 17 anos, sendo $43,5 \%(n=10)$ do sexo masculino e $56,5 \%(n=13)$ do sexo feminino.

Quanto ao tipo de epilepsia, oito pacientes $(34,7 \%)$ tinham epilepsia do tipo síndrome de Lennox-Gastaut, três pacientes eram do tipo sintomático e cinco do tipo criptogênico; dez pacientes tinham epilepsia focal sintomática $(43,5 \%)$; quatro pacientes, epilepsia focal criptogênica (17,4\%); e um paciente apresentou epilepsia não-classificada (4,4\%).

Todos faziam uso de politerapia, sendo a associação mais adotada valproato e benzodiazepínico.

Dos 23 pacientes que iniciaram a dieta, dois desistiram no primeiro mês, pois as mães não quiseram mantê-la; seis permaneceram por seis meses, pois o controle das crises não atingiu a 
expectativa dos pais e houve abandono após esse período; 15 mantiveram a dieta por um período de 12 meses.

Foram assinalados obstipação em 4 pacientes $(17,4 \%)$ e sonolência em 11 pacientes $(47,8 \%)$, no início do estudo; náuseas e vômitos em 10 pacientes (43,4\%); epistaxe em 1 paciente
(4,3\%); infecções de repetição em 3 pacientes $(13,0 \%)$ e recusa alimentar em 3 pacientes $(13,0 \%)$.

Não se observaram mudanças significativas no perfil metabólico da dosagem sérica de potássio, cloro, creatinina, cálcio, proteína total, albumina, gama proteína e ácido úrico (Tabelas 1

Tabela 1. Medidas descritivas das variáveis estudadas, em cada momento de avaliação, de pacientes com epilepsia, submetidos a dieta cetogência, aderentes e desistentes (antes da dieta e um mês pós-dieta). UNIFESP-EPM, São Paulo, 2004.

\begin{tabular}{|c|c|c|c|c|c|c|}
\hline \multirow{2}{*}{ Variáveis } & & \multicolumn{2}{|c|}{ Desistentes $(n=8)$} & \multicolumn{2}{|c|}{ Aderentes $(n=15)$} & \\
\hline & & Média & DP & Média & DP & \\
\hline \multirow[t]{3}{*}{ Sódio (meq/l) } & Antes & 136,17 & 0,75 & 137,93 & 0,88 & \\
\hline & 1 mês & 136,83 & 0,75 & 138,13 & 0,74 & $p^{*}=0,004$ \\
\hline & & & $p \#=0,001$ & & & $p \S=0,029$ \\
\hline \multirow[t]{3}{*}{ Potássio (meq/l) } & Antes & 4,02 & 0,16 & 4,06 & 0,23 & \\
\hline & 1 mês & 3,97 & 0,14 & 4,08 & 0,19 & $p^{*}=0,999$ \\
\hline & & & $p \#=0,551$ & & & $p \S=0,999$ \\
\hline \multirow[t]{3}{*}{ Cloro (mmol/l) } & Antes & 102,83 & 0,98 & 102,07 & 1,39 & \\
\hline & 1 mês & 102,83 & 0,75 & 101,93 & 0,96 & $p^{*}=0,765$ \\
\hline & & & $p \#=0,351$ & & & $p \S=0,983$ \\
\hline \multirow[t]{3}{*}{ Uréia (mg/dl) } & Antes & 18,00 & 0,89 & 18,13 & 0,92 & \\
\hline & 1 mês & 19,00 & 0,89 & 18,40 & 0,83 & $p^{*}=0,041$ \\
\hline & & & $p \#=0,648$ & & & $p \S=0,182$ \\
\hline \multirow[t]{3}{*}{ Creatinina (mg/dl) } & Antes & 0,68 & 0,08 & 0,70 & 0,07 & \\
\hline & 1 mês & 0,68 & 0,08 & 0,69 & 0,09 & $p^{*}=0,576$ \\
\hline & & & $p \#=0,401$ & & & $p \S=0,068$ \\
\hline \multirow[t]{3}{*}{ Cálcio (mg/dl) } & Antes & 9,90 & 0,28 & 9,90 & 0,16 & \\
\hline & 1 mês & 9,90 & 0,22 & 9,83 & 0,18 & $p^{*}=0,074$ \\
\hline & & & $p \#=0,990$ & & & $p \S=0,512$ \\
\hline \multirow[t]{3}{*}{ Glicemia (mg/dl) } & Antes & 73,83 & 1,94 & 75,87 & 2,7 & \\
\hline & 1 mês & 73,17 & 1,17 & 74,33 & 2,8 & $p^{*}=0,001$ \\
\hline & & & $p \#=0,299$ & & & $p \S=0,709$ \\
\hline \multirow[t]{3}{*}{ Pt (g/dl) } & Antes & 6,85 & 0,19 & 6,90 & 0,15 & \\
\hline & 1 mês & 6,87 & 0,12 & 6,85 & 0,15 & $p^{*}=0,258$ \\
\hline & & & $p \#=0,740$ & & & $p \S=0,596$ \\
\hline \multirow[t]{3}{*}{ Ácido úrico (mg/dl) } & Antes & 3,12 & 0,91 & 3,97 & 1,44 & \\
\hline & 1 mês & 3,25 & 0,90 & 4,59 & 1,50 & $p^{*}=0,338$ \\
\hline & & & $p \#=0,586$ & & & $p \S=0,785$ \\
\hline \multirow[t]{3}{*}{$\mathrm{Col}(\mathrm{mg} / \mathrm{dl})$} & Antes & 148,00 & 41,30 & 165,00 & 38,21 & \\
\hline & 1 mês & 181,00 & 57,91 & 195,87 & 63,13 & $p^{*}=0,015$ \\
\hline & & & $p \#=0,188$ & & & $p \S=0,128$ \\
\hline \multirow[t]{3}{*}{ Tri (mg/dl) } & Antes & 82,67 & 31,61 & 71,33 & 26,75 & \\
\hline & 1 mês & 109,50 & 59,24 & 93,13 & 30,18 & $p^{*}=0,001$ \\
\hline & & & $p \#=0,805$ & & & $p \S=0,293$ \\
\hline \multirow[t]{3}{*}{$\mathrm{Bic}(\mathrm{mmol} / \mathrm{l})$} & Antes & 22,50 & 1,05 & 24,31 & 1,61 & \\
\hline & 1 mês & 21,90 & 1,48 & 22,91 & 1,43 & $p^{*}=0,006$ \\
\hline & & & $p \#=0,805$ & & & $p \S=0,982$ \\
\hline
\end{tabular}

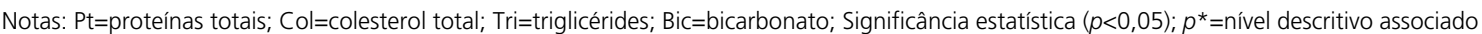
à comparação entre as médias obtidas nos instantes "antes" e "1 mês"; $p \S=$ nível descritivo associado ao efeito de interação, ou seja, se a

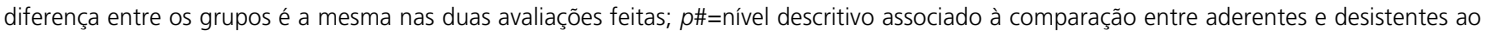
longo do tempo. Independentemente do grupo, houve aumento significante nas variáveis triglicérides, colesterol e uréia. Independentemente do grupo, houve redução significante nas variáveis bicarbonato e glicemia. Houve aumento significante em relação ao sódio. O grupo dos desistentes apresentou um aumento mais evidente do que os aderentes. Para as demais variáveis, nenhuma diferença foi detectada. 
e 2). Houve aumento significante em relação ao sódio, o grupo de desistentes mostrou um aumento mais evidente do que os aderentes entre o nível pré-dieta e um mês pós-dieta. Notou-se aumento significante do colesterol total, dos triglicérides e da uréia entre o nível pré-dieta e um mês pós-dieta (Tabela 1). Além disso, registrou-se redução significante do bicarbonato sérico e da glicemia entre o nível pré-dieta e um mês pós-dieta (Tabela 1). Porém, esses níveis não saíram da normalidade (Tabela 1). Houve tendência à normalização do perfil metabólico, no decorrer do período de estudo (Tabela 2).

Observou-se controle total das crises epilépticas em três pacientes, muito bom controle (>90\%) em cinco, bom controle (50\%-90\%) em seis, controle regular $(<50 \%)$ em sete e ausência de efeito em dois (Tabela 1).

\section{DISCUSS Ã O}

Os benefícios da dieta cetogênica são bem documentados ${ }^{8}$. Muitos estudos fazem referência a observações médicas e a complicações psicossociais, porém poucos mencionam as complicações da dieta.

Os efeitos indesejados da dieta cetogênica relacionam-se, em geral, com a intolerância à dieta, como náuseas, vômitos, diarréia ou mesmo obstipação intestinal. Pode ocorrer, ainda, sonolência no começo do tratamento, recomendando-se a retirada de drogas antiepilépticas potencialmente sedativas, ao iniciar a dieta citada ${ }^{8,9}$. No acompanhamento, percebeu-se sonolência, provocada, provavelmente, pelo efeito aditivo da dieta cetogênica com drogas sedativas, apenas no início do tratamento em 11 pacientes (47,8\%), os quais melhoraram muito com a descontinuidade de drogas sedativas.

A constipação decorre da diminuição do volume ingerido e de fibras. Essas queixas ocorreram com 4 pacientes $(17,4 \%)$, tendo obtido melhora no quadro com a introdução de fibras nos primeiros 3 meses. Ainda no princípio da dieta, devido aos níveis elevados de corpos cetônicos,
Tabela 2. Medidas descritivas das variáveis estudadas, de pacientes com epilepsia, submetidos a dieta cetogência, aderentes e desistentes, Unifesp-EPM, São Paulo, 2004.

\begin{tabular}{|c|c|c|c|c|c|}
\hline \multirow{2}{*}{ Variáveis } & \multicolumn{2}{|c|}{ Desistentes $(n=8)$} & \multicolumn{3}{|c|}{ Aderentes $(n=15)$} \\
\hline & Média & DP & Média & DP & \\
\hline $\begin{array}{l}\text { Sódio } \\
\text { antes } \\
12 \text { meses }\end{array}$ & 136,17 & 0,75 & $\begin{array}{l}137,93 \\
138,27\end{array}$ & $\begin{array}{l}0,88 \\
0,80\end{array}$ & $p^{*}=0,610$ \\
\hline $\begin{array}{l}\mathbf{K} \\
\text { antes } \\
12 \text { meses }\end{array}$ & 4,02 & $\begin{array}{c}t=0,830 \\
0,16 \\
\#=0,240\end{array}$ & $\begin{array}{l}4,06 \\
3,95\end{array}$ & $\begin{array}{l}0,23 \\
0,09\end{array}$ & $p^{*}=0,587$ \\
\hline $\begin{array}{l}\mathbf{C l} \\
\text { antes } \\
12 \text { meses }\end{array}$ & 102,83 & $\begin{array}{c}0,98 \\
t=0,060\end{array}$ & $\begin{array}{l}102,07 \\
101,20\end{array}$ & $\begin{array}{l}1,39 \\
1,01\end{array}$ & $p^{*}=0,381$ \\
\hline $\begin{array}{l}\mathbf{U} \\
\text { antes } \\
12 \text { meses }\end{array}$ & 18,00 & $\begin{array}{c}0,89 \\
\neq=0,314\end{array}$ & $\begin{array}{l}18,13 \\
19,00\end{array}$ & $\begin{array}{l}0,92 \\
0,85\end{array}$ & $p^{*}=0,804$ \\
\hline $\begin{array}{l}\text { C } \\
\text { antes } \\
12 \text { meses }\end{array}$ & 0,68 & $\begin{array}{c}0,08 \\
t=0,518\end{array}$ & $\begin{array}{l}0,70 \\
0,66\end{array}$ & $\begin{array}{l}0,07 \\
0,07\end{array}$ & $p^{*}=0,825$ \\
\hline $\begin{array}{l}\mathbf{C a} \\
\text { antes } \\
12 \text { meses }\end{array}$ & 9,90 & $\begin{array}{l}0,28 \\
\neq=0,498\end{array}$ & $\begin{array}{l}9,90 \\
9,77\end{array}$ & $\begin{array}{l}0,16 \\
0,09\end{array}$ & $p^{*}=0,218$ \\
\hline $\begin{array}{l}\text { Gli } \\
\text { antes } \\
12 \text { meses }\end{array}$ & 73,83 & $\begin{aligned} & 1,94 \\
= & 0,123\end{aligned}$ & $\begin{array}{l}75,87 \\
73,93\end{array}$ & $\begin{array}{l}2,70 \\
2,25\end{array}$ & $p^{*}=0,149$ \\
\hline $\begin{array}{l}\mathbf{P t} \\
\text { antes } \\
12 \text { meses }\end{array}$ & 6,85 & $\begin{array}{l}0,19 \\
=0,877\end{array}$ & $\begin{array}{l}6,90 \\
6,81\end{array}$ & $\begin{array}{l}0,15 \\
0,12\end{array}$ & $p^{*}=0,514$ \\
\hline $\begin{array}{l}\text { Ac. úrico } \\
\text { antes } \\
12 \text { meses }\end{array}$ & 3,12 & $\begin{array}{l}0,91 \\
\neq=0,059\end{array}$ & $\begin{array}{l}3,97 \\
3,84\end{array}$ & $\begin{array}{l}1,44 \\
1,11\end{array}$ & $p^{*}=0,446$ \\
\hline $\begin{array}{l}\text { Col } \\
\text { antes } \\
12 \text { meses }\end{array}$ & 148 & $\begin{array}{l}41,30 \\
t=0,646\end{array}$ & $\begin{array}{l}165,00 \\
158,93\end{array}$ & $\begin{array}{l}38,21 \\
25,00\end{array}$ & $p^{*}=0,429$ \\
\hline $\begin{array}{l}\text { Tri } \\
\text { antes } \\
12 \text { meses }\end{array}$ & 82,67 & $\begin{array}{l}31,61 \\
t=0,278\end{array}$ & $\begin{array}{l}71,33 \\
72,07\end{array}$ & $\begin{array}{l}26,75 \\
22,04\end{array}$ & $p^{*}=0,364$ \\
\hline $\begin{array}{l}\text { Bic } \\
\text { antes } \\
12 \text { meses }\end{array}$ & 22,5 & $\begin{aligned} & 1,05 \\
= & 0,171\end{aligned}$ & $\begin{array}{l}24,31 \\
23,74\end{array}$ & $\begin{array}{l}1,61 \\
1,33\end{array}$ & $p^{*}=0,144$ \\
\hline
\end{tabular}

Notas: Sódio (meq/l) K: potássio (meq/l); Cl: cloro (mmol/l); U: uréia (mg/dl); C: creatinina (mg/dl); Ca: cálcio $(\mathrm{mg} / \mathrm{dll}) ;$ Gli: glicemia $(\mathrm{mg} / \mathrm{dl})$; Pt: proteínas totais (g/dl); Ac.úrico: Ácido úrico (mg/dl); Col: colesterol total $(\mathrm{mg} / \mathrm{dl})$; Tri: triglicérides (mg/dl); Bic: bicarbonato $(\mathrm{mmol} / \mathrm{l})$; $p^{*}=$ nível descritivo associado à comparação entre as médias obtidas ao longo do acompanhamento; $p \#=$ nível descritivo associado à comparação entre aderentes e desistentes ao longo do tempo; Não houve diferença significante nos momentos estudados; Não houve diferença significante entre desistentes e aderentes. 
podem aparecer náuseas e vômitos, que costumam melhorar em 4 a 7 dias. No presente estudo, por ocasião da implantação da dieta, notou-se a presença de náuseas e vômitos em 10 pacientes $(43,4 \%)$, que melhoraram com a adaptação e com a introdução de suco de laranja.

Cálculo renal constitui uma complicação conhecida da dieta cetogênica, entretanto a exata incidência da formação de cálculos nesses pacientes é desconhecida ${ }^{10}$. A alta porcentagem da formação da pedra por urato continua não explicada, contudo pode ser associada à acidúria e subseqüente $\mathrm{pH}$ ácido urinário, ou à restrição hídrica $^{11-13}$. Não observamos hipercalcemia e hipercalciúria, tendo o controle ultrassonográfico permanecido normal, sem indicar a formação de cálculos em todos os pacientes, como também não assinalaram acidose metabólica sintomática, que necessitasse de correção.

Outro efeito indesejável observado consiste na presença de infecções intercorrentes, como conseqüência da alteração da função dos granulócitos, levando a um maior risco de infecções ${ }^{14}$. Três dos pacientes acompanhados (13\%) sofreram infecções intercorrentes nos primeiros quatro meses de dieta. Após esse período, houve uma normalização do número de infecções.

A dieta cetogênica pode provocar um aumento no teor de colesterol e de triglicérides $^{15-17}$. Constatou-se mudança do colesterol total e de triglicérides entre o nível pré-dieta e um mês pós-dieta (Tabela 1). Depois desse período, houve queda do colesterol total e de triglicérides, no decorrer do tratamento, atingindo os níveis mensurados antes da dieta em $90 \%$ dos pacientes. Houve, ainda, alteração do perfil metabólico do sódio, da glicemia e da uréia, entre o nível pré-dieta e um mês pós-dieta, mantendo a faixa da normalidade e assim permanecendo no decorrer do estudo. Hematoma de pele é um efeito colateral que tem sido discutido por famílias de pacientes, principalmente nos primeiros três meses da dieta, podendo persistir pelo tempo de tratamento $^{18}$. Durante a pesquisa, um paciente começou a apresentar epistaxe com três meses da introdução da dieta. Foi realizada avaliação hematológica e não se verificou alteração, tendo a epistaxe desaparecido em dois meses, não havendo necessidade de interrupção da dieta.

Em todos os pacientes assistidos, no início da dieta, introduziu-se a suplementação de vitaminas e sulfato ferroso, e não se observaram sinais clínicos de avitaminoses.

Enquanto a dieta típica ocidental consiste em grande proporção (50\% ou mais) de carboidratos, na dieta cetogênica a proporção de carboidratos cai para $5 \%$, sendo $90 \%$ formada basicamente por gorduras. Neste estudo, três pacientes ganharam peso nos primeiros três meses de dieta, devido ao seu estado nutricional prévio inadequado, nos demais pacientes que iniciaram a dieta com peso acima da média observou-se uma queda do peso, após esse período inicial observou-se crescimento pôndero estatural linear esperado para a idade, confirmando as anotações relatadas em outros trabalhos na literatura' ${ }^{19-21}$.

Segundo vários estudos, de um terço a metade dos pacientes consegue obter uma excelente resposta, em termos de redução acentuada ou até de completo controle das crises, o outro um terço das crianças tem uma resposta parcial ou incompleta resposta, com alguma redução na freqüência e gravidade das crises, e um terço não tem benefício com a dieta 8,9,21,22. Neste estudo, 3 pacientes ficaram livres de crises (13,0\%); 5 tiveram muito bom controle de crises, mais do que $90,0 \%$ das crises controladas $(21,7 \%) ; 6(26 \%)$ tiveram um bom controle das crises, 50,0\% a 90,0\%; em 7 pacientes $(30,4 \%$ ) o controle foi regular, menor do que $50,0 \%$ das crises; em 2 pacientes (8,7\%) não se observou efeito na freqüência de crises.

Em vista dos resultados, a equipe multidisciplinar considerou a dieta efetiva e bem tolerada, apesar das limitações.

\section{CONCLUSÃO}

Conclui-se que a dieta cetogênica pode constituir-se em uma alternativa eficaz para o tratamento de epilepsia refratária na infância, entretanto deve-se ter consciência dos potenciais 
efeitos adversos e detectá-los precocemente. Essas alterações são bastante raras e, na maioria das vezes, podem ser prevenidas, sem necessidade de interromper a dieta. A dieta clássica e a modificada são efetivas, mas impõem restrição dietética rígida.

Antes de optar por essa forma de tratamento, é imprescindível contar com a cooperação dos pais. A instalação dessa dieta requer o treinamento de uma equipe multidisciplinar que irá estabelecer, em etapas, a adequação da dieta para os padrões psicológicos, sociais, nutricionais e culturais de cada família, acompanhando e orientando o paciente até a conclusão da intervenção.

\section{REFERÊ N CIAS}

1. Galván-Manso M, Arellano M, Sans A, Sanmarti FX, Gómez L. Dieta cetogénica: una alternativa válida en epilepsias refractarias. Rev Neurol. 2001; 33(11):1010-4.

2. Stafstrom C, Spencer S. The ketogenic diet: a therapy in search of an explanation. Neurology. 2000; 54(2):282-3.

3. Nordli DR. The ketogenic diet. Uses and abuses. Neurology. 2002; 58(Suppl 7):S21-4.

4. Cuéllar R, Molinero M. Tratamiento de los niños com epilepsia de difícil control. Rev Neurol. 2003; 37(4):371-5.

5. Commission on Classification and Terminology of the International League Against Epilepsy. Proposal for revised clinical and electroencephalographic classification of epileptic seizures. Epilepsia. 1981; 22(4):489-501.

6. Freeman JM, Kelly MT, Freeman JB. The epilepsy diet treatment. An introduction to The Ketogenic Diet. New York: Demos Vermande; 1996. p.24-7.

7. Huttenlocher PR. Ketonaemia and seizures: metabolic and anticonvulsant effects of the two ketogenic diets in childhood epilepsy. Pediatr Res. 1976; 10(5):536-40.

8. Freeman J, Veggiotti P, Lanzi G, Tagliabue A, Perucca E. The ketogenic diet: from molecular mechanisms to clinical effects. Epilepsy Res. 2006; 68(2): 145-80.

9. Forcadas-Berdusán MI. Indicaciones y resultados de los tratamientos no farmacológicos de las epilepsias: estimulación vagal, dieta cetógena y rayos gamma. Rev Neurol. 2002; 35(Supl 1): s144-50.
10. Kielb S, Koo HP, Bloom DA, Faerber GJ. Nephrolithiasis associated with the ketogenic diet. J Urol. 2000; 164(2):464-6.

11. Freitas A, Valente KD, Casella EB, Paz JÁ, Marques-Dias MJ. Ketogenic diet: ten year experience in children. J Epilepsy Clin Neurophysiol. 2002; 8(2):91.

12. Hemingway C, Freeman JM, Pillas DJ, Pyzik PL. The ketogenic diet: A 3- to 6- year follow-up of 150 children enrolled prospectively. Pediatrics. 2001; 108(4):898-905.

13. Kossoff EH, Pyzik PL, Furth SL, Hladkly HD, Freeman JM, Vining EPG. Kidney stones, carbonic anhydrase inhibitors, and the ketogenic diet. Epilepsia. 2002; 43(10):1168-71.

14. Panico LR, Demartini MG, Rios VG, Carniello MA. Dieta cetogénica en la epilepsia refractaria infantil: respuesta electroclínica, complicaciones y efectos secundarios. Rev Neurol. 2000; 31(3):212-20.

15. Marsh EB, Freeman JM, Kossoff EH, Vining EPG, Rubenstein JE, Pyzik PL, et al. The outcome of children with intractable seizures: a 3- to 6- year follow-up of 67 children who remained on the ketogenic diet less than one year. Epilepsia. 2006; 47(2):425-30.

16. Rios VG, Panico LR, Demartini MG, Carniello MA. Complicaciones en el tratamiento de la epilepsia con dieta cetogênica. Rev Neurol. 2001; 33(10): 909-15.

17. Rubenstein JE, Kossoff EH, Pyzik PL, Vining EPG, McGrogan JR, Freeman JM. Experience in the use of the ketogenic diet as early therapy. J Child Neurol. 2005; 20(1):31-4.

18. Berry-Kravis E, Booth G, Taylor A, Valentino LA. Bruising and the ketogenic diet: Evidence for diet-induced changes in platelet function. Ann Neurol. 2001; 49(1):98-103.

19. Nordli DR, Kuroda MM, Carrol J, et al. Experience with the ketogenic diet in infants. Pediatrics. 2001; 108(1):129-33.

20. Vining EPG, Pyzik P, McGrogan J, Hladky H, Anand A, Kriegler S, et al. Growth of children on the ketogenic diet. Dev Med Child Neurol. 2002; 44(12):796-802.

21. Peterson SJ, Tangney CC, Pimentel-Zablah EM, Hjelmgren B, Booth G, Berry-Kravis E. Changes in growth and seizure reduction in children on the ketogenic diet as a treatment for intractable epilepsy. J Am Diet Assoc. 2005; 105(5):718-25.

22. Thiele EA. Assessing the efficacy of antiepileptic treatments: The ketogenic diet. Epilepsia. 2003; 44(Suppl 7):26-9.

Recebido em: 21/9/2004

Versão final reapresentada em: 27/4/2006 Aprovado em: 9/8/2006 